\title{
On the Existence and Computation of Minimum Attention Optimal Control Laws
}

\author{
Pilhwa Lee and F.C. Park, Fellow
}

\begin{abstract}
One means of capturing the cost of control implementation of a general nonlinear control system is via Brockett's minimum attention criterion, defined as a multidimensional integral of the rate of change of the control with respect to state and time. Although shown to be important in human motor control and robotics applications, a practical difficulty with this criterion is that the existence of solutions is not always assured; even when they exist, obtaining local solutions numerically is difficult. In this paper we prove that, for the class of controls consisting of the sum of a time-varying feedforward term and a time-varying feedback term linear in the state, existence of a suboptimal solution can be guaranteed. We also derive a provably convergent gradient descent algorithm for obtaining a local solution, by appealing to the Liouville equation representation of a nonlinear control system and adapting iterative methods originally developed for boundary flow control. Our methodology is illustrated with a two degree-of-freedom planar robot example.
\end{abstract}

Index Terms-Minimum attention, optimal control, Liouville equation, boundary flow control

\section{INTRODUCTION}

Given a general nonlinear system $\dot{x}=f(x, u, t)$ evolving on the state space $\mathcal{X}$, where $x \in \mathcal{X} \subseteq \mathbb{R}^{n}$ is the state and $u \in$ $\mathcal{U} \subseteq \mathbb{R}^{m}$ is the control, Brockett [1] proposes the following functional-referred to as the minimum attention criterionas a measure of the cost of implementation of a control law $u=u(x, t)$ :

$$
J(u)=\frac{1}{2} \int_{0}^{T} \int_{\mathcal{X}}\left\|\frac{\partial u}{\partial x}\right\|^{2}+\left\|\frac{\partial u}{\partial t}\right\|^{2} d x d t .
$$

The basic premise behind this criterion is that the simplest control law to implement is a constant input: the more frequently a control changes, the more resources are required to implement it. Since control laws typically depend on both the state and time, their cost of implementation can be linked with the rate at which the control varies with respect to changes in state - the first term of (1) - and changes in time - the second term of (1).

Some intriguing connections have been pointed out between the attention criterion and existing theories for human motor control and learning [2], [3], [4], [5], [6], [7], [18], [19], [20]. For example, the minimum attention control paradigm offers a generative explanatory model for human motor skill learning, in which skills that are initially reliant on feedback gradually become more and more open loop with practice,

Pilhwa Lee (lead author) is with the Department of Mathematics, Morgan State University, Baltimore, MD, USA, pilhwa.lee@morgan.edu.

F.C. Park (corresponding author) is with the Department of Mechanical Engineering, Seoul National University, Seoul, Korea, fcp@snu.ac.kr.

Manuscript received Nov 22, 2019; revised January 20, 2021. e.g., perfecting a golf swing. At the same time, the minimum attention principle can also explain how, in tasks that rely on sensory feedback like catching a ball, the reliance on visual feedback is initially low, but steadily increases and peaks immediately before the ball is caught (we address this example further in our experiments). New insights and perspectives on the control of soft robots have also been obtained via the minimum attention paradigm [8].

The main challenge in forging the minimum attention paradigm into a practical control methodology is that solutions to the associated multidimensional variational problem for (1) are difficult to come by; even the existence of solutions is not always guaranteed in the general case.

One way to make this problem more tractable is to restrict attention to systems of the form $\dot{x}=f(x)+G(x) u$, with $G(x) \in \mathbb{R}^{n \times m}$ given and $u$ of the form $u(x, t)=K(t) x+v(t)$, where $K(t) \in \mathbb{R}^{m \times n}$ is a feedback gain matrix and $v(t) \in \mathbb{R}^{m}$ is a feedforward term. For this class of controls, some results on minimum attention trajectories have been obtained for simple kinematic models of wheeled mobile robots [9]. Additional assumptions on the attention functional, such as setting the terminal cost to be the mean-square state error and weighting the attention functional probabilistically, make it possible to approximate the problem as a linear quadratic regulator (LQR), for which efficient solutions are readily obtainable [10], [2]. By and large, however, the minimum attention paradigm has been effectively applied only in limited cases, with only approximate solutions obtained under restricted settings.

In this paper we expand the class of problems for which minimum attention control laws can be meaningfully framed, in the sense of guaranteeing the existence of solutions and obtaining them numerically. The main contributions are (i) a proof of the existence of a minimum attention control under the assumption of a control of the form $u(x, t)=K(t) x+v(t)$ over a bounded state space, with $K(t)$ and $v(t)$ twice continuously differentiable, and (ii) an iterative gradient descent algorithm for computing this minimum attention control law.

The key ideas that we draw upon are the Liouville partial differential equation (PDE) representation for nonlinear systems as proposed by Brockett [11], and the observation that the existence question for the minimum attention problem is structurally similar to the well-posedness problem for boundary flow control in the Navier-Stokes equations [12]. Specifically, [12] proves the existence of a solution minimizing the rate of change in the control with respect to space and time for a twodimensional boundary fluid flow, while tracking prescribed fluid flow velocities governed by the Navier-Stokes equations. We show that the minimum attention control problem posed in the Liouville PDE setting is, despite some differences 
(e.g., the state space for the minimum attention problem is of arbitrary dimension $n$ rather than two-dimensional, and a control over the entire state space is sought rather than only on the boundary), structurally similar to the flow control problem addressed in [12].

Addressing these differences, we show that the one-shot iterative method developed in [12] can be generalized to the minimum attention problem. Specifically, an iterative Monte Carlo-based gradient descent method is developed to find local solutions to the minimum attention problem, whose convergence to a local minimum can also be guaranteed via ellipticity (specifically, boundedness of the second-order variation of the associated Lagrangian).

The remainder of the paper is organized as follows. Section II formulates the minimum attention problem in a Liouville PDE setting and makes explicit the structural similarities and differences with the flow control problem of [12]. Section III proves the existence of a solution for the class of controls considered in this paper, and describes the iterative gradient descent method together with a proof of its convergence to a local solution. Section IV provides examples of minimum attention control laws obtained for a two-link planar robot.

\section{PROBLEM Formulation}

Given the first-order nonlinear system

$$
\dot{x}=f(x, u, t)
$$

where $x \in \mathcal{X} \subseteq \mathbb{R}^{n}$ is the state, $u \in \mathcal{U} \subseteq \mathbb{R}^{m}$ is the control, and $f$ is continuously differentiable with respect to $x$ and $u$, the standard optimal control problem for (2) is usually formulated as seeking the optimal $u^{*}$ that minimizes the criterion

$$
\phi(x(T))+\int_{0}^{T} L(x, u) d t,
$$

subject to boundary conditions on the initial and terminal states and other equality and inequality constraints imposed on the state and control. In practical situations one may also have to contend with a distribution of initial states rather than a single initial state. In [11] the case is made that by replacing (2) by the Liouville partial differential equation

$$
\frac{\partial \rho(x, t)}{\partial t}=-\left\langle\frac{\partial}{\partial x}, f(x, u) \rho(x, t)\right\rangle,
$$

where $\rho(x, t)$ is the probability density for $x$ with boundary condition $\rho(x, 0)=\rho_{0}(x)$, then not only can an initial distribution of states be naturally included, but the criterion (3) can also be expanded to include, e.g., non-trajectory dependent terms such as the last two terms in

$$
\begin{aligned}
\eta= & \int_{\mathcal{X}} \rho(x, T) \phi(x) d x+\int_{0}^{T} \int_{\mathcal{X}} \rho(x, t) L(x, u) d x d t \\
& +\frac{1}{2} \int_{\mathcal{X}}\left\|\frac{\partial u}{\partial x}\right\|^{2} d x+\frac{1}{2} \int_{0}^{T}\left\|\frac{\partial u}{\partial t}\right\|^{2} d t .
\end{aligned}
$$

There is in fact a natural stochastic optimal control interpretation to optimizing (5) subject to (4): Equation (4) can be identified with the Fokker-Planck equation corresponding to the stochastic differential equation $d x=f(x, u) d t+G d w$ (with $G$ assumed zero); given a probability density $\rho(x, 0)=\rho_{0}(x)$ at $t=0, \rho(x, t)$ then describes the time evolution of the density for $x$.

Adopting the Liouville equation representation, the minimum attention optimal control problem can now be formulated as follows. Given a desired terminal density $\psi(x)$, define the attention functional (for convenience we henceforth omit the terminal $\phi(x(T))$ term from our functional) $\eta$ as

$$
\begin{aligned}
\eta= & \int_{\mathcal{X}}(\rho(x, T)-\psi(x))^{2} d x \\
& +\int_{\mathcal{X}} \int_{0}^{T}\left(\left\|\frac{\partial u}{\partial x}\right\|^{2}+\left\|\frac{\partial u}{\partial t}\right\|^{2}\right) d t d x,
\end{aligned}
$$

in which the first and second terms of $\eta$ can be identified as the terminal cost and running cost, respectively. In this setting the objective is to seek the $u(x, t)$ that minimizes the attention functional (6) while driving the probability density governed by (4) from $\rho(x, 0)=\rho_{0}(x)$ at $t=0$ to $\rho(x, T)=\psi(x)$ at $t=T$.

Equations (4) and (6) can be compared to the boundary velocity control problem in [12], in which the Navier-Stokes equations and the associated cost functional are as follows:

$$
\begin{aligned}
& \frac{\partial v}{\partial t}+(v \cdot \nabla) v-\nu \Delta v+\nabla p=0 \text { in }(0, T) \times \Omega, \\
& \nabla \cdot v=0 \quad \text { in }(0, T) \times \Omega, \\
& v=u \quad \text { on }(0, T) \times \Gamma_{c} \\
& v=0 \quad \text { on }(0, T) \times\left(\Gamma \backslash \Gamma_{c}\right),
\end{aligned}
$$

where $v(x, t)$ is a two-dimensional flow over the time interval $[0, T]$ in the bounded domain $\Omega$ with boundary $\Gamma, \Gamma_{c} \subset \Gamma$ denotes the region over which the control is valid, $p$ and $\nu$ respectively denote the hydrodynamic pressure and kinematic viscosity of the fluid, and $\nabla$ and $\Delta$ respectively the gradient and Laplacian operators. The initial velocity $v(x, 0)=v_{0}(x)$ is given, and the boundary velocity control, denoted by $u$, is required to satisfy the compatibility conditions

$$
\int_{\Gamma_{c}}\langle u, n\rangle d x=0,
$$

where $n$ denotes the unit outward normal vector on $\Gamma$, and

$$
\left.u\right|_{t=0}=\left.v_{0}\right|_{\Gamma_{c}} .
$$

The associated objective is to find a boundary control $u$ and a velocity field $v$ such that the cost functional

$$
\begin{aligned}
\mathcal{J}(v, u) & =\int_{0}^{T} \int_{\Gamma_{c}}\left(\|u\|^{2}+\alpha\left\|\frac{\partial u}{\partial t}\right\|^{2}+\alpha\left\|\frac{\partial u}{\partial x}\right\|^{2}\right) d x d t \\
& +\int_{0}^{T} \int_{\Omega}\|v-V\|^{2} d x d t
\end{aligned}
$$

is minimized subject to $v$ and $u$ satisfying (7)-(8) with the prescribed fluid velocity $V$ and the regularizing parameter $\alpha$.

Although the minimum attention problem seeks a control over the entire domain rather than on the boundary, and the domain is also of arbitrary dimension $n$ rather than two-dimensional, the one-shot method used in [12] for the boundary flow control problem can be generalized to prove both existence of a solution and convergence of an iterative gradient descent algorithm for the minimum attention problem. 
As a first step, the first-order necessary conditions for (6) subject to the state dynamics (4) can be derived explicitly in terms of the augmented Lagrangian $\mathscr{L}$ :

$\mathscr{L}=\eta-\int_{\mathcal{X}} \int_{0}^{T} \lambda\left(\frac{\partial \rho(x, t)}{\partial t}+\left\langle\frac{\partial}{\partial x}, f(x, u) \rho(x, t)\right\rangle\right) d t d x$,

where $\lambda$ denotes the Lagrange multiplier. Then $\frac{\delta \mathscr{L}}{\delta \lambda}=0$ leads to the state equations (4), while the adjoint equation $\frac{\delta \mathscr{L}}{\delta \rho}=0$ leads to

$$
\frac{\partial \lambda(x, t)}{\partial t}+\left\langle\frac{\partial \lambda(x, t)}{\partial x}, f(x, u)\right\rangle=0
$$

subject to the boundary condition $\lambda=\rho-\psi$ at $t=T$. Finally, the optimality condition $\frac{\delta \mathscr{L}}{\delta u}=0$ leads to

$$
\Delta u+\frac{\partial^{2} u}{\partial t^{2}}=\frac{\partial f^{T}}{\partial u} \frac{\partial \lambda}{\partial x} \rho .
$$

Equations (4), (12), (13) together constitute the first-order necessary conditions for the optimal solution $(\rho, u, \lambda)$. Since $\lambda$ is conserved along the direction of advection $f$, the Lagrange multiplier $\lambda(x(t), t)$ along solutions to (2) can be expressed in terms of the terminal densities $\rho$ and $\psi$ as

$$
\begin{aligned}
\lambda(x(t), t) & =\lambda\left(x(t)+\int_{t}^{T} f d t, T\right) \\
& =\rho\left(x(t)+\int_{t}^{T} f d t, T\right)-\psi\left(x(t)+\int_{t}^{T} f d t\right) \\
& =\rho(x(T), T)-\psi(x(T)) .
\end{aligned}
$$

We make use of these first-order necessary conditions in the next section.

\section{MAIN RESULT}

We now assume that the control $u$ is of the form

$$
u=K(t) x+v(t),
$$

where $K(t) \in \mathbb{R}^{m \times n}$ and $v(t) \in \mathbb{R}^{m}$ are both $C^{2}([0, T])$, i.e., twice continuously differentiable on $[0, T]$.

Proposition 1. Given an initial density $\rho_{0}(x)$ and terminal density $\psi(x)$ over $\mathcal{X}$ bounded, there exists a sequence $\left(K_{i}, v_{i}\right)$, with $K_{i}(t) \in \mathbb{R}^{m \times n}$ and $v_{i}(t) \in \mathbb{R}^{m}$ both $C^{2}([0, T])$ and equicontinuous, that uniformly converges to some $(\hat{K}, \hat{v})$ in the space $C^{2}([0, T])$, such that $\hat{u}=\hat{K}(t) x+\hat{v}(t)$ minimizes the functional $\eta$ in (6).

Proof. By continuity $K$ and $v$ are both bounded. $\eta$ is also bounded from above and below, i.e., $\eta_{\mathrm{m}} \leq \eta \leq \eta_{\mathrm{M}}$ where

$$
\begin{aligned}
& \eta_{\mathrm{m}}=\inf _{K, v \in C^{2}([0, T])} \eta(K, v), \\
& \eta_{\mathrm{M}}=\sup _{K, v \in C^{2}([0, T])} \eta(K, v) .
\end{aligned}
$$

Let $K_{n}$ and $v_{n}$ be minimizing sequences for $\eta$, i.e.,

$$
\lim _{n \rightarrow \infty} \eta\left(K_{n}, v_{n}\right)=\eta_{\mathrm{m}},
$$

equicontinuous in the class of $C^{2}([0, T])$. Since both $K_{n}$ and $v_{n}$ are pointwise bounded and equicontinuous, there exists a subsequence $\left(K_{m}, v_{m}\right)$ in $C^{2}([0, T])$ uniformly converging to $(\hat{K}, \hat{v})[13] .(\hat{K}, \hat{v})$ and the corresponding $\hat{u}$ minimize the functional $\eta$ of (6).

\section{Iterative Algorithm}

Having established the existence of a solution, we now develop an iterative gradient descent algorithm (Algorithm 1) to solve the first-order necessary conditions for the optimal $(K(t), v(t))$. The control $u$ in the form of (15) is substituted into the optimality condition (13). Keeping in mind that solutions obey (2), the explicit equation to be solved is, after some calculation,

$$
\begin{array}{r}
(\ddot{K}+K B \dot{K}) x(t)=\rho(x(t), t) B^{T} \frac{\partial \lambda(x(t), t)}{\partial x} \\
-\ddot{v}-2 \dot{K} f-K(A f+B K f+B \dot{v}),
\end{array}
$$

where $A=\frac{\partial f}{\partial x}, B=\frac{\partial f}{\partial u}$, and $x(t)$ is governed by (2) with initial condition $x(0)=x_{0}$. The associated $\rho(x, t)$ follows the Liouville equation (4) with initial condition $\rho(x, 0)=\rho_{0}(x)$ while $\lambda(x(t), t)$ is obtained from (14).

Referring to Algorithm 1, for initialization we generate an initial reference trajectory and control $\left(x^{*}, u^{*}\right)$ using, e.g., the linear quadratic regulator method of [14]. The initial timevarying feedback gain and feedforward $\left(K^{(0)}(t), v^{(0)}(t)\right)$ are then derived from the linearized optimal control perturbed from $\left(x^{*}, u^{*}\right)[2]$ :

$$
u(x, t)=u^{*}(t)-R^{-1} B(t)^{T} P(t)\left(x-x^{*}(t)\right),
$$

where $R \in \mathbb{R}^{m \times m}$ is a symmetric positive-definite matrix, and $P(t) \in \mathbb{R}^{n \times n}$ is generated backward from the matrix Riccati differential equation

$$
-\dot{P}=P A+A^{T} P-P B R^{-1} B^{T} P,
$$

with terminal boundary condition $P(T)=P_{\mathrm{f}} . K^{(0)}$ and $v^{(0)}$ are prescribed as follows:

$$
\begin{aligned}
K^{(0)} & =-R^{-1} B^{T} P, \\
v^{(0)} & =u^{*}+R^{-1} B^{T} P x^{*} .
\end{aligned}
$$

Given $K^{(0)}(t)$ and $v^{(0)}(t)$ from the optimal control, we solve for the density distribution $\rho^{(0)}(x, t)$ and evaluate the cost $\eta^{(0)}$.

We note that our algorithm does not require the initial path to satisfy the terminal endpoint constraint. For initialization of the line search parameter $\epsilon_{(m)}^{(n)}$, we set $\epsilon_{(0)}^{(0)}=\epsilon_{0}$, where $n$ and $m$ respectively indicate the iterations for the outer loop control update and inner loop line search.

We now show that if the second variation of $f$ with respect to $u$ is degenerate, our algorithm converges to a local minimizer for any initial control:

Proposition 2. Algorithm 1 converges to a local minimizer for any initial control if the second variation of $f$ with respect to $u$ is degenerate.

Proof. Following [12], let $\mathcal{X}$ be a Hilbert space with norm $\|\cdot\|$ and let $\mathscr{L}$ be a $C^{2}$ functional on $\mathcal{X}$. Suppose $\hat{u}$ is a local minimizer for $\mathscr{L}$ close to the initial control, and let $B$ be a ball in $\mathcal{X}$ centered at $\hat{u}$ with radius sufficiently large so as to include the initial control. From the optimality condition (13),

$$
\frac{\delta \mathscr{L}}{\delta u}=-\int_{\mathcal{X}} \int_{0}^{T}\left(\Delta u+\frac{\partial^{2} u}{\partial t^{2}}-\left(\frac{\partial f}{\partial u}\right)^{T} \frac{\partial \lambda}{\partial x} \rho\right) d t d x .
$$


If the second variation of $f$ with respect to $u$ is degenerate, for all $\tilde{u} \in B$ and for all variations $h_{1}, h_{2} \in X$ of $\tilde{u}$, from (11) the second variation of $\mathscr{L}$ can be written

$$
\frac{\delta^{2} \mathscr{L}}{\delta u^{2}}(\tilde{u})\left(h_{1}, h_{2}\right)=-\int_{\mathcal{X}} \int_{0}^{T}\left(\Delta h_{2}+\frac{\partial^{2} h_{2}}{\partial t^{2}}\right) \cdot h_{1} d t d x
$$

where $\frac{\delta^{2} \mathscr{L}}{\delta u^{2}}(\tilde{u})\left(h_{1}, h_{2}\right)$ is the bilinear form related to the second derivatives of $\mathscr{L}$. Because $h_{2}$ is a variation of $\tilde{u}$ that is linear in $x$ as assumed in (15), $\nabla^{2} h_{2}$ is degenerate. Integrating by parts,

$$
\int_{0}^{T} \frac{\partial^{2} h_{2}}{\partial t^{2}} \cdot h_{1} d t=-\int_{0}^{T} \frac{\partial h_{2}}{\partial t} \cdot \frac{\partial h_{1}}{\partial t} d t
$$

Taking the time derivative of $h=K \delta x$ as a variation of $\tilde{u}$,

$$
\frac{\partial h}{\partial t}=(\dot{K}+K A) \delta x \text {. }
$$

Define the constants $c_{1}$ and $c_{2}$ to be the supremum and infimum of the ratio $\left\|\frac{\partial h}{\partial t}\right\| /\|h\|$, i.e.,

$$
c_{1}=\sup _{K \delta x \neq 0} \frac{\|(\dot{K}+K A) \delta x\|}{\|K \delta x\|}, \quad c_{2}=\inf _{K \delta x \neq 0} \frac{\|(\dot{K}+K A) \delta x\|}{\|K \delta x\|} .
$$

Because $K, \dot{K}$, and $A$ are all continuous and $\delta x$ is bounded in $\mathcal{X}$, the existence of a positive finite $c_{1}$ and $c_{2}$ are guaranteed. The second variation of $\frac{\delta^{2} \mathscr{L}}{\delta u^{2}}\left(h_{1}, h_{2}\right)$ is therefore bounded from above and below, i.e.,

$$
\begin{aligned}
& \frac{\delta^{2} \mathscr{L}}{\delta u^{2}}(\tilde{u})\left(h_{1}, h_{2}\right) \leq c_{1}^{2} T\|\mathcal{X}\|\left\|h_{1}\right\|\left\|h_{2}\right\| \\
& \frac{\delta^{2} \mathscr{L}}{\delta u^{2}}(\tilde{u})\left(h_{1}, h_{1}\right) \geq c_{2}^{2} T\|\mathcal{X}\|\left\|h_{1}\right\|^{2},
\end{aligned}
$$

satisfying ellipticity of the Lagrangian. It is now possible to choose a positive set of parameters $\epsilon_{k}<\left(2 c_{2}^{2} / c_{1}^{2}\right)$ such that the iteration

$$
u(k+1)=u(k)-\epsilon_{k} \nabla \mathscr{L}(u(k)), k=0,1,2, \ldots
$$

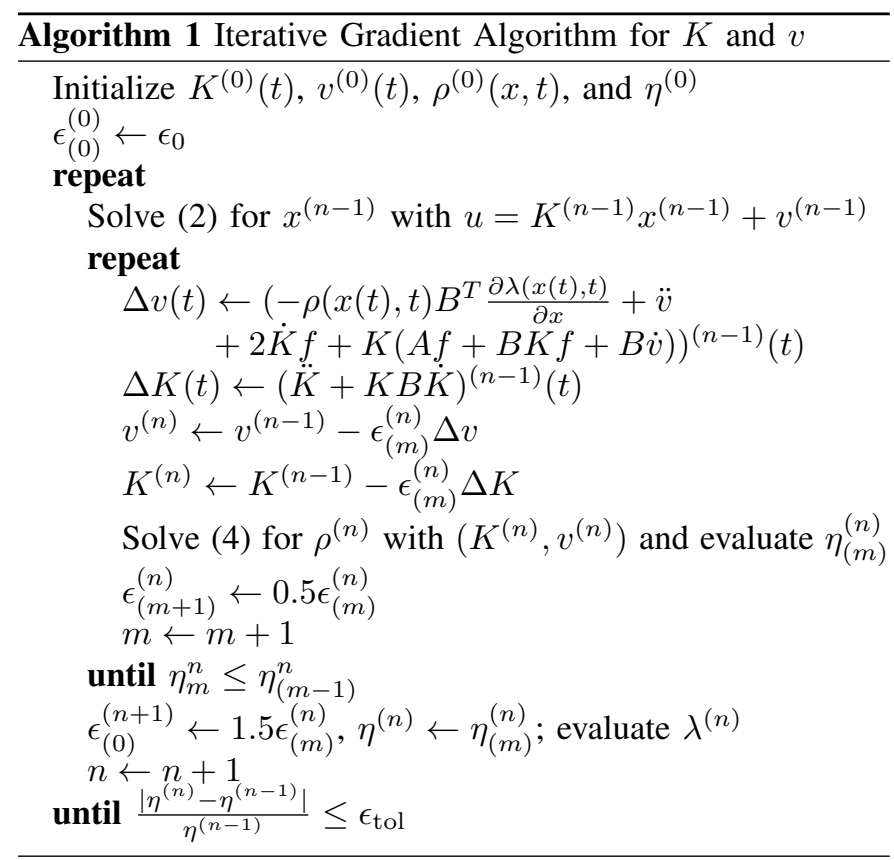

converges to $\hat{u}$ for any initial iterate $u(0) \in B$. For further related details see [15].

\section{EXAMPLE}

As an example, we derive the minimum attention control law for a two-link planar robot arm. The dynamic equations are of the form

$$
\tau=M(q) \ddot{q}+b(q, \dot{q})
$$

where $q \in \mathbb{R}^{2}$ represents the joint angle vector and $\dot{q} \in \mathbb{R}^{2}$ its velocity, $\tau \in \mathbb{R}^{2}$ is the joint torque vector, $M(q) \in \mathbb{R}^{2 \times 2}$ is the symmetric positive-definite mass matrix, and $b(q, \dot{q}) \in$ $\mathbb{R}^{2}$ consists of the Coriolis and gravity terms. Expressions for $M(q)$ and $b(q, \dot{q})$ can be found in Appendix A. Define the state vector $x=(q, \dot{q}) \in \mathbb{R}^{4}$ and the control $u=\tau \in \mathbb{R}^{2}$. The dynamic equations in state space form then become

$$
\dot{x}=f(x, u)=\left[\begin{array}{c}
x_{2} \\
M^{-1}(x)\left(u-b\left(x_{1}, x_{2}\right)\right)
\end{array}\right] .
$$

In this example, $u=K(t) x+v(t)$ is bounded with $K(t)$ and $v(t)$ continuous in $[0, T]$. Since $f=f(x, u)$ is continuously differentiable, $(q, \dot{q})$ is bounded in its trajectory, and and the second variation of $f$ with respect to $u$ is degenerate, our minimum attention algorithm is applicable.

We perform two sets of experiments. In the first experiment, the objective is to drive the system from the initial state $x_{\text {init }}=(0,0,0,0)$ to the end-effector position-velocity $\phi_{\mathrm{f}}=(-0.26,0.40,0,0)$ at terminal time $T=0.5 \mathrm{sec}$ (the forward kinematics $\phi(x)$ is described in Appendix B). In the second experiment we drive the system from the initial state $x_{\text {init }}=(0.1,-0.1,0,0)$ to the end-effector positionvelocity $\phi_{\mathrm{f}}=(-0.32,0.27,0,0)$ at terminal time $T=0.5$ sec. The associated initial distribution $\rho_{0}(x)$ is prescribed to be centered at $x_{\text {init }}$, with compact support over eight grids using a smoothed Dirac delta function (see [16]). The attention cost (6) for this example is chosen to be

$$
\begin{aligned}
\eta & =\gamma \int_{\mathcal{X}}\left\|\phi(x)-\phi_{\mathrm{f}}\right\|^{2} \rho(x, T) d x \\
& +\int_{\mathcal{X}} \int_{0}^{T}\left\|\frac{\partial u}{\partial x}\right\|^{2}+\left\|\frac{\partial u}{\partial t}\right\|^{2} d t d x .
\end{aligned}
$$

The adjoint boundary condition is $\lambda=\gamma\left\|\phi(x)-\phi_{\mathrm{f}}\right\|^{2}$ at $t=T$, with $\lambda$ at $(x(t), t)$ derived similarly to (14):

$$
\lambda(x(t), t)=\gamma\left\|\phi(x(T))-\phi_{\mathrm{f}}\right\|^{2} .
$$

For our example we choose $\gamma=10^{6}$. The state space $\mathcal{X}$ is taken to be $[-5,5] \times[-5,5] \times[-300,300] \times[-300,300]$, discretized into 256 uniform intervals over each dimension, while the time domain is discretized into 40 intervals.

For Experiment 1, the initial $K^{(0)}(t)$ and $v^{(0)}(t)$ are obtained from (22) and (23) with $P_{\mathrm{f}}=\operatorname{diag}\left(10^{5}, 1,10^{5}, 1\right)$ and $R=\operatorname{diag}(0.4,1.3565)$. These $K^{(0)}(t)$ and $v^{(0)}(t)$ are also used for Experiment 2 with the same set of parameters. For the derivatives of $K$ and $v$, second-order centered differencing is used except for the initial and terminal points at $t=0$ and $t=T$, where first-order differencing is employed. The second derivatives of $K$ and $v$ are similarly computed from their first derivatives. We set $\epsilon_{0}=2.0 \times 10^{-3}$ and $\epsilon_{\text {tol }}=5.0 \times 10^{-5}$ in the 


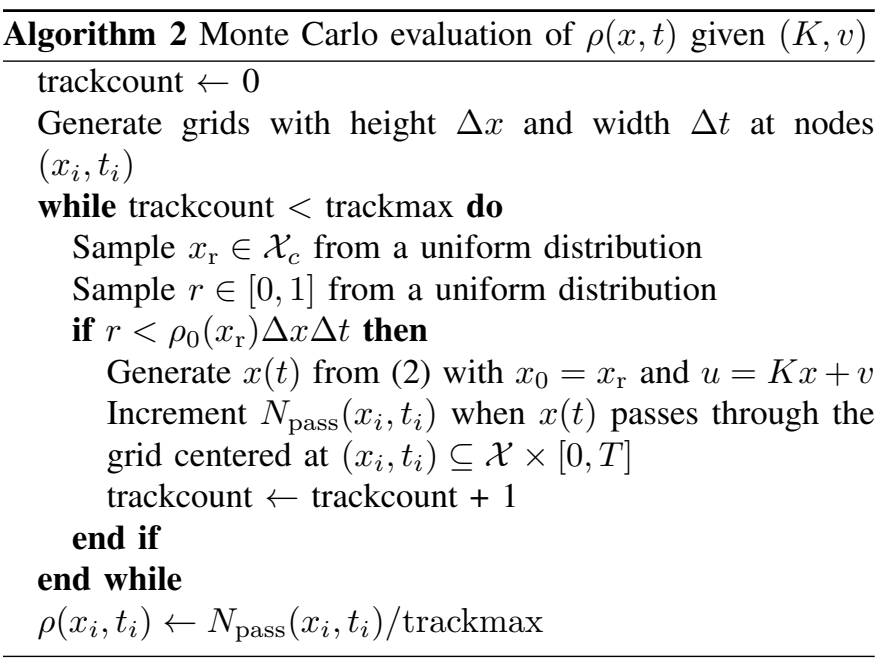

main algorithm. Also, at each iteration of the main algorithm, for the evaluation of $\rho^{(i)}(x, t)$ we use the basic Monte Carlo Algorithm 2 [17] (but other methods are also possible).

Figure 1(a) shows the results of Experiment 1, in which the initial path in $q_{1}-q_{2}$ space is shown in red, and the minimum attention path is shown in blue. Note that although the initial path does not reach the target goal state, our algorithm converges to a final path that reaches the target. Figure 1(b) shows snapshots of the robot arm motion as it traverses the minimum attention path from the initial to final pose. Figure 2 shows trajectories for the minimum attention control $u(t)$, feedback gain $K(t)$, and feedforward term $v(t)$. Consistent with observations from the human motor control literature [2], [18], [19], [20], we also observe that the feedforward term initially dominates in the early part of the motion but eventually approaches zero, while the feedback term initially starts out small but rapidly dominates toward the latter part of the motion, when feedback is used for precise positioning. The attention cost plotted against the number of iterations is shown in Figure 3.

Results of Experiment 2 are shown in Figure 4, which shows the initial and final paths in $q_{1}-q_{2}$ and the corresponding arm pose snapshots for a different pair of initial and terminal states. The optimal trajectories obtained for $u(t), K(t)$, and $v(t)$ also show similar characteristics to those for Experiment 1, i.e., a smooth transition from feedforward to feedback terms as the motion progresses from the initial to final state.

\section{CONClusion}

Using the Liouville partial differential equation representation for nonlinear systems [11], in this paper we have provided a proof of the existence of a minimum attention control as formulated in [1] under the assumption of a control of the form $u(x, t)=K(t) x+v(t)$. Exploiting the structural similarity between this problem and a boundary flow control problem involving the Navier-Stokes equations, we adopt the one-shot method of [12] to develop an iterative gradient algorithm for the numerical solution of the minimum attention control. An example involving a two-link planar robot arm is used to illustrate the algorithm, with results that bear close similarity
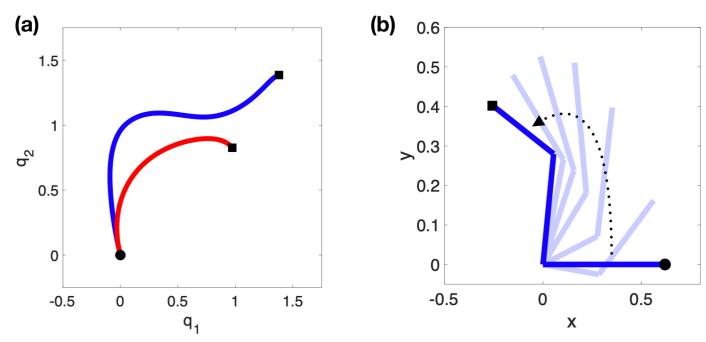

Fig. 1. Experiment 1: the robot is driven from the initial configuration $x_{\text {init }}=$ $(0,0,0,0)$ to the end-effector position-velocity $\phi_{\mathrm{f}}=(-0.26,0.40,0,0)$ at terminal time $T=0.5 \mathrm{sec}$. (a) Initialized (red), converged (blue) paths in $q_{1}-q_{2}$ with the initial state (circle) and the final state (box). (b) A sequence of arm pose snapshots for the final converged motion. The initial and final hand positions are indicated by the circle and box, respectively.
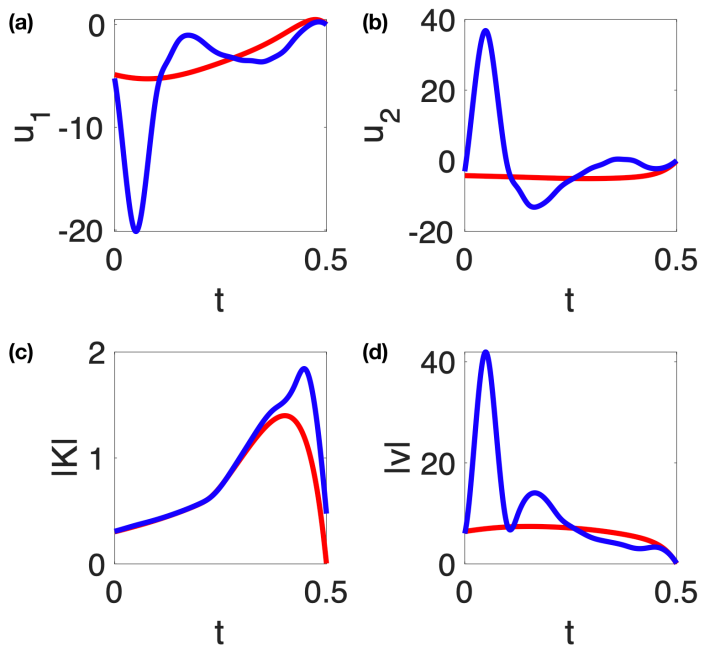

Fig. 2. Experiment 1: Graphs of (a) $u_{1}(t)$; (b) $u_{2}(t)$; (c) $\|K(t)\|$; (d) $\|v(t)\|$. Initial trajectories are indicated in red, while converged trajectories are indicated in blue.

to observed human arm reaching movements that transition from feedforward to feedback control as the goal state is reached. Our algorithm, although not yet sufficiently efficient for real-time control applications, reliably converges to a local minimum for a wide range of initial conditions. The possibility of using our algorithm to generate training data for, e.g., a reinforcement learning-based method for motion control [21]

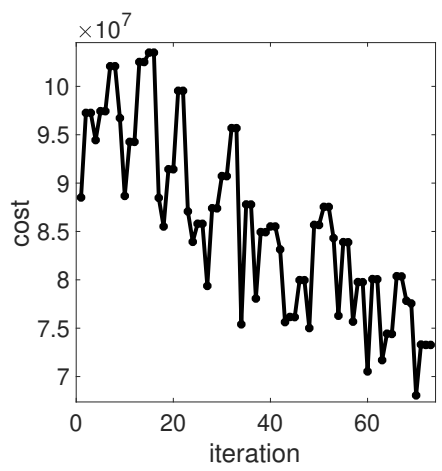

Fig. 3. Experiment 1: Attention cost $\eta$ plotted against the number of iterations. 

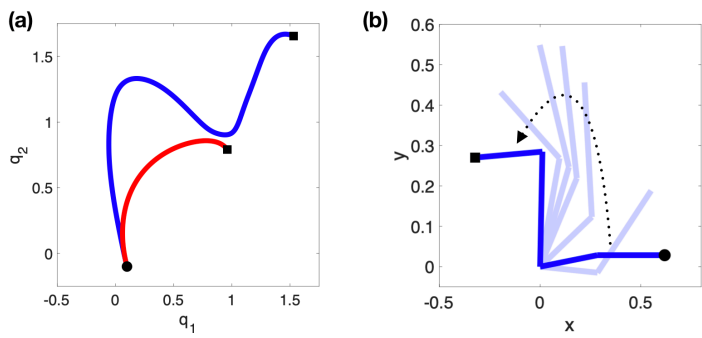

Fig. 4. Experiment 2: the robot is driven from the initial configuration $x_{\text {init }}=(0.1,-0.1,0,0)$ to the end-effector position-velocity $\phi_{\mathrm{f}}=$ $(-0.32,0.27,0,0)$ at terminal time $T=0.5 \mathrm{sec}$. (a) Initialized (red), converged (blue) paths in $q_{1}-q_{2}$ with the initial state (circle) and the final state (box). (b) A sequence of arm pose snapshots for the final converged motion. The initial and final hand positions are indicated by the circle and box, respectively.

is but one possible practical application.

\section{ACKNOWLEDGMENTS}

F.C. Park was supported in part by SNU SRRC grant NRF2016R1A5A1938472, SNU BMRR grant DAPAUD190018ID, the Naver Labs AMBIDEX Project, SNU BK21+ Program in Mechanical Engineering, SNU-IAMD, and the SNU Institute for Engineering Research.

\section{APPENDIX A}

\section{DYNAMICS OF TWO-LINK ROBOT ARM}

Elements of the mass matrix $M(q)$ and bias terms $b(q, \dot{q})$ are as follows [7]:

$$
\begin{aligned}
m_{11}= & I_{1}+I_{2}+2 M_{2} L_{1} S_{2} \cos q_{2}+M_{2} L_{1}^{2} \\
m_{22}= & I_{2} \\
m_{12}= & I_{2}+M_{2} L_{1} S_{2} \cos q_{2} \\
m_{21}= & m_{12} \\
b_{1}= & -M_{2} L_{1} S_{2}\left(2 \dot{q}_{1}+\dot{q}_{2}\right) q_{2} \sin q_{2}+B_{11} \dot{q}_{1}+B_{12} \dot{q}_{2} \\
& +g\left[\left(M_{1} S_{1}+M_{2} L_{1}\right) \sin q_{1}+M_{1} S_{2} \sin \left(q_{1}+q_{2}\right)\right] \\
b_{2}= & M_{2} L_{1} S_{2} \dot{q}_{1}^{2} \sin q_{2}+B_{22} \dot{q}_{2}+B_{21} \dot{q}_{1} \\
& +g M_{2} S_{2} \sin \left(q_{1}+q_{2}\right),
\end{aligned}
$$

where $g$ denotes gravity, and $I_{i}, M_{i}, L_{i}, S_{i}$ respectively denote the inertia, mass, arm length, and center of mass of link $i$.

\section{APPENDIX B FORWARD KINEMATICS}

The forward kinematics map $\phi(x)$ maps the joint positionvelocity pair $x=(q, \dot{q})$ to the end-effector Cartesian position $(X, Y)$ and velocity $(\dot{X}, \dot{Y})$ via

$$
\left(\begin{array}{c}
X \\
Y \\
\dot{X} \\
\dot{Y}
\end{array}\right)=\left(\begin{array}{c}
L_{1} \cos q_{1}+L_{2} \cos \left(q_{1}+q_{2}\right) \\
L_{1} \sin q_{1}+L_{2} \sin \left(q_{1}+q_{2}\right) \\
-L_{1} \dot{q}_{1} \sin q_{1}-L_{2}\left(\dot{q}_{1}+\dot{q}_{2}\right) \sin \left(q_{1}+q_{2}\right) \\
L_{1} \dot{q}_{2} \cos q_{1}+L_{2}\left(\dot{q}_{1}+\dot{q}_{2}\right) \cos \left(q_{1}+q_{2}\right)
\end{array}\right) .
$$

\section{REFERENCES}

[1] R.W. Brockett, "Minimum attention control," Proc. 36th Conf. Decis. Control, pp. 2628-2632, 1997.

[2] C. Jang, J. Lee, S. Lee, and F.C. Park, "A minimum attention control law for ball catching," Bioinspir. and Biomim., vol. 10, no. 5, 2015.

[3] E. Todorov and M.I. Jordan, "Optimal feedback control as a theory of motor coordination," Nat. Neurosci., vol. 5, no. 11, pp. 1226-1235, 2002.

[4] S. Schaal, "Learning control in robotics," IEEE Robot. Autom. Mag., vol. 17, no. 2, pp. 20-29, 2010.

[5] D.M. Wolpert, J. Diedrichsen, and J.R. Flanagan, "Principles of sensorimotor learning," Nat. Rev. Neurosci., vol. 12, no. 12, pp. 739-751, 2011.

[6] A. Battaglia-Mayer and R. Caminiti, "Corticocortical systems underlying high-order motor control," J Neurosci., vol. 39, no. 23, pp. 4404-4421, 2019.

[7] E. Nakano, H. Imamizu, R. Osu, Y. Uno, H. Gomi, T. Yoshioka, and M. Kawato, "Quantitative examinations of internal representations for arm trajectory planning: minimum commanded torque change model," $J$ Neurophysiol., vol. 81, no. 5, pp. 2140-2155, 1999.

[8] C.D. Santina, M. Bianchi, G. Grioli, F. Angelini, M. Catalano, M. Garabini, and A. Bicchi, "Controlling soft robots: balancing feedfback and feedforward elements," IEEE Robot. Autom. Mag., vol. 24, no. 3, pp. 75-83, 2017.

[9] S. Lee and F.C. Park, "Mobile robot motion primitives that take into account the cost of control," in Latest Advances in Robot Kinematics, J. Lenarcic and M. Husty, Eds., pp. 429-436, Heidelberg: Springer, 2012.

[10] R.W. Brockett, "Minimizing attention in a motion control context," Proc. 42th Conf. Decis. Control, vol. 4, pp. 3349-3352, 2004.

[11] R.W. Brockett, Chapter 2. Notes on the control of the Liouville equation, in Control of Partial Differential Equations, Lecture Notes inMath., vol. 2048, Berlin: Springer, pp. 101-129, 2012.

[12] M.D. Gunzburger and S. Manservisi, The velocity tracking problem for Navier-Stokes flows with boundary control, SIAM J Control. Optim., vol. 39, no. 2, pp. 594-634, 2000

[13] W. Rudin, Principles of Mathematical Analysis, Third Ed., McGrawHill, 1976.

[14] W. Li and E. Todorov, Iterative linear quadratic regulator design for nonlinear biological movement systems, Proc. 1st Int. Conf. Informatics in Control, Autom, Robotics, pp. 222-229, 2004.

[15] P.G. Ciarlet, Introduction to Numerical Linear Algebra and Optimisation. Cambridge, UK: Cambridge Univ. Press, 1989.

[16] C.S. Peskin, The immersed boundary method, Acta Numerica, vol. 11, pp. 479-517, 2002

[17] G. Bird, Molecular Gas Dynamics and the Direct Simulation of Gas Flows. Oxford Univ. Press, 1994.

[18] M. Kawato, Internal models for motor control and trajectory planning, Curr. Opin. Neurobiol., vol. 9, pp. 718-727, 1999.

[19] D.M. Wolpert, Z. Ghahramani, and J.R. Flanagan, Perspectives and problems in motor learning, Trends. Cogn. Sci., vol. 5, pp. 487-494, 2001.

[20] D. Liu and E. Todorov, Evidence for the flexible sensorimotor strategies predicted by optimal feedback control, J. Neurosci., vol. 27, pp. 93549368, 2007.

[21] S. Levine, C. Finn, T. Darrell, and P. Abbeel, End-to-end training of deep visuomotor policies, J. Mach. Learn. Res., vol. 17, pp. 1-40, 2016. 